Information Gathered Mainly From Perbal Statements of Prospector Masch. 2946

CAPY NOME PRECINCP

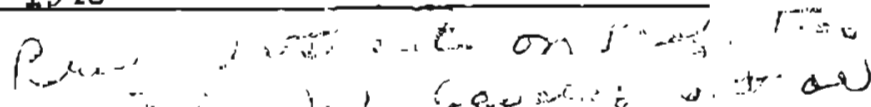

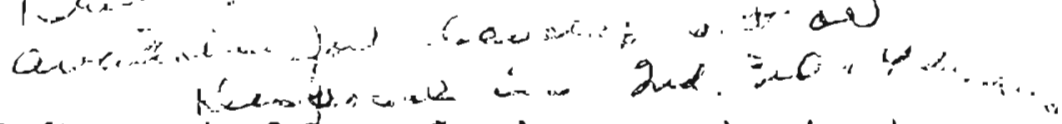

R. W. Silver, Nome - The only good ground left open in Solonon-Casadopaga region is at head of Solomon Ryver - undredged above ingget Creek, owned by bank could be worked with bullazer.

Ruby Creek, tributary Casadepaga Rtver. Was worked with horses and scraper. Bulldozer proposition for future.

Penolope Creok. Nlison omed ground, aled. Longloy had property - may have dropped it by now. Young mas shoveled in $\$ 8: 00$ a day thero sometime

R. O. Lee, Solomon River D1gtrict. Right 11 mit mouth of Myatery Creak. Old bonch has been drifted. Lee Bros, have the ground. Propet to lark that and up the cresk. Eydraulic and 1 1/2 cu. ft. bucket dredge.

West Creok - Lee Bros. expect to lease ground from prosed owners. Will put $1 \mathrm{l} / 2 \mathrm{cu}$. $\mathrm{ft}$. dredge on creak. Several miles could be dredged thu with benches.

Bill Munz, Nome - Copper, lead and allver lode. For examination. Location not divalged.

Small cooper vein below Gugtafson-Swodman 1944 camp on Hungry Crook, tribatery of Oregon Crack, tributary of Cripole River.

St. Lawrence I sland - S. W. Capo - Gold lodo and placer - Richard Okturkyak. Froe gold viatble in quartz.

Broen - Gold-antimony and S11scovitch gold-antimony. Two lode probpecta - noar tranbortation - good posibilities. Head of Snake and Nome River.

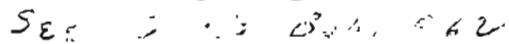




\section{COUNCIL OITY PRECIRCT}

Fred Durocher, in Fish Biver beain - Prospects on Net Creek which heada ouposite head of Rock Crook, tributary of Aggie Croek. Splanev Bros. are aaid to have found prospecti opposite head of Aggio on Cacho Creek.

Dredge on $\#$, Aggle Creek, September, 1944. Probably will not go above \$4 as 4 and 5 were llght. Good pay on $\$ 7$ (and 6). May be bulldozer and hydraulic posibility.

Etchepuk River - Raklmo found good prosoect there below foothilla. Durocher gays formation is good and he will drill.

Camo Creek, tributary Mubutulik River, throe miles above Admiral. Durocher shoveled in

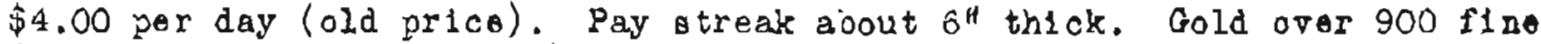
(orobably small pay atreak).

Clydo Glass found copoer, lood and silver minerals on Melsing Oroek. Also geveral pear ghaped metcoriteg. Gless ataked two bench claims on Blkhorn Crook, tributary of Nlukluk above Camp Creok. Claims really ataked for quartz lodo croesed by dredge. Bstimates vein $20^{\prime}$ wide - large amounts of froe gold tr quartz found in dredge concentrateg and on talling piles bolowthts rein. Sot visiblo in place.

Copper, zinc, lead lode - southeast of Mt. Bendoleben. Apparently omall. Investigated by Thorye, U. S. B. of Mi nee.

(1) Heriert Jemes, Solomon flektmo. Gold, sllver, lead, 1ron veln - throe crooks oouth of Onilak Silver Mine. Hideat part of vein - 16'. Voln traced conoldorablo distance uo ridge and found on opposite s1de of ridge on general line of atriko.

Tubutulik River - (Bill Geleler, Nome - gold property below mouth of Caro, (Bome abovo), and on tributary to Tubutulik in viclaity of Camp Croek. Could have been sold for $\$ 250,000$ ( 9 ) and Cleary Hill in there al so. Jobn Largon, Mike Acheff (Nomi) have been tbere. Bench on rlght limit of Camp Croek contains good gold values.) (Camp Creek misnemed on map).

Fred Durocher. Near Camp Croek, tributary to Thbutulik, there is dredging ground; erook 1 tgelf too small. F. D. mede $\$ 4.00$ por day including dead work. Uppor end of Tubutulik Canyon ( 1 ) - 5 to 6 mlles abovo mouth of Camp Creak and up to Death Valley are prospecte. Gold dredging ground in Tubutulik Ritrer. (placer tin ldentified by miner.)

In creok 10 miles from Aggie Croek, tributary Blsh Bivor, thero aro good gold prospecto. May be Cacho Croek.

Jones Pup - right limit tributary of Mubutulik on lower ond of Death Palloy. Worth looking into for gold. 


\section{$-2-$}

COUNCIL CITY PRECINOT (Cont'd)

Charley Blatchford, Some - Supposed to know about mica." Worked for Thorne. 
TFRTITORY OF ALASKA

Department of Mineg

Informat1 on Gathered Mainly From Verbal Statements of Prospoctors March. 1946

\section{FAIRHAVEN PRECINCT}

Inmachuk River Bagin - Bunkhouse Johnston - write J. H. Johnston, Doering. Small bench prospocts on Milroy Croek - narrow - small bulldozer and hydraulic operation if anything. Once worked by "rormation Jack" MeCartney who also saloed on Fortune Creek.

Johnston bench channel, Cunningham-1944, $30^{\prime}$ wide, $3^{\prime}$ gravel, $8^{\prime}$ muck, frozen, 85 per oq. It. In drift. Length not determined.

Botween J. H. Johnston's lower camp on Gannum and mouth of Milroy Croek - 200' wide right lintt bench channel, 35 to 404 per fard whor prospected. Pockets mined by hand at varlous places on Hannum Creok. Staked by Johnston and Firenze from Johngton lower cabin to mouth of Cunningham; also all or nost of Cunntagham. Cunnlngham Creek ground sluiced in oarly days for fow hundred foet below mouth of harris Creek.

Keenan once vigltod place and thought oreot too small for him. Lameredallied on Cunningham - drill hole within 10 of benoh channel later found by Johnston. Lamers missed Hannum benches.

Lower half of Hannum jetween Milroy and Cunningham thought to be better than upper half. Mostly rlght linit bench.

At nouth of Bonanza Crook, tributary Good Hope, (Jaok) Hagendorn Ild took out duno containing $\$ 5,000$ eccording to Johnston and Hienze.

Johnston hag left limt bench clatim on the Inmachuk River a shore distance abore upper Dry Croek which he bolieves are worksblo.

Dry Creok Dredging Company expects to beable to work much of Inmachul perbaps oven abov mouth of Bannum Creok and up Pinneil Biver. Woithor of latter have boen prospected sroperly.

Upper Inmachuk - Colling, Hannum, Cunnlngham, Milroy (soe Ray Mendenhall), atc. (According to Paddy Hatt, galena is in closely apaced narrow otringers in Milroy oreeir cut). Placer gold - drifting 1943 on gome. Other headvater tributariog of Inmachuk are of interest.

Comiseloner R. L. Gill1,, Candle - At one time small atamped to Koopuk Craok, tributary of lower Bucklend (about $10 \mathrm{miles}$ from mouth 1 ). Belimos still ontpe thore. Creek comes in below old Buckland Village. Don Moto? A Japtuged to bring gold from Lucky Star Crosk, tributary to lagoon near mouth of Buckland River. Stzean orobably hoad in game area as Koopuk Creok.

Jumy Creok, tributary of Candlo Creok, tributary of Kiwalik River. Billy French and

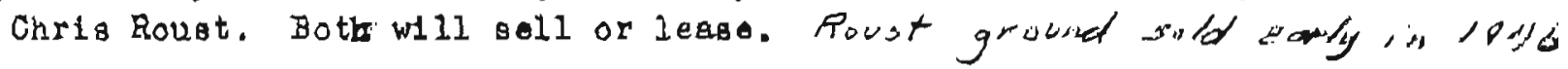




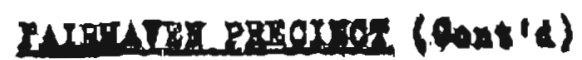

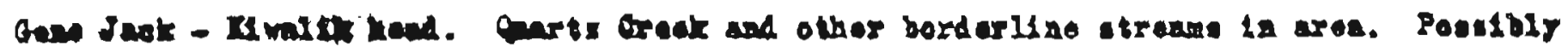

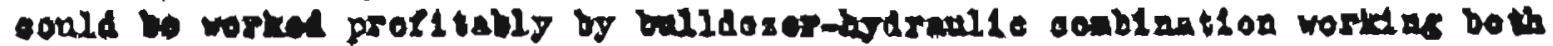

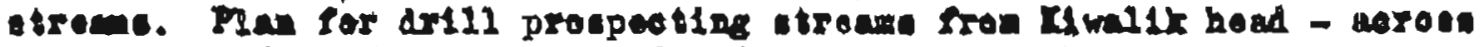

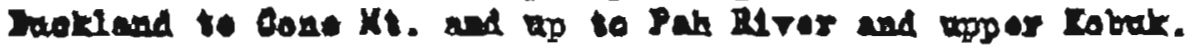

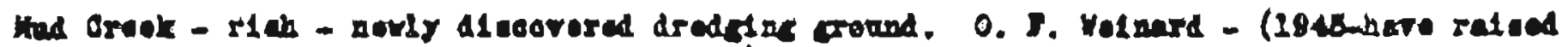
mones and WII work themedres).

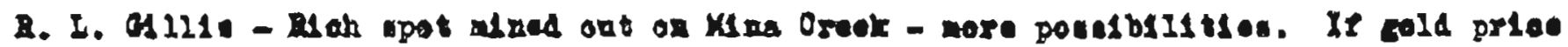

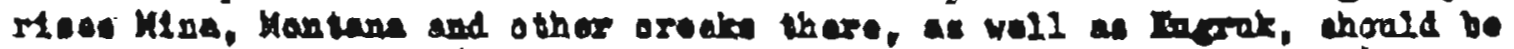

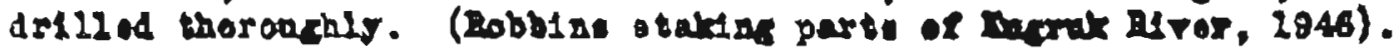

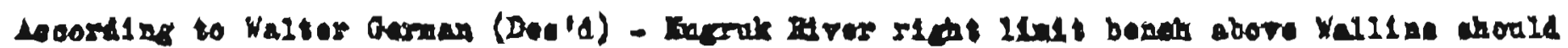
be proproted.

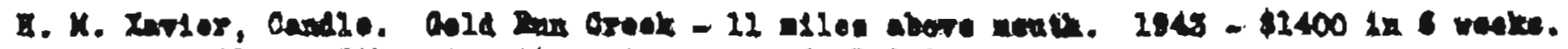

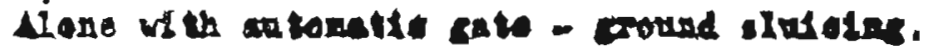

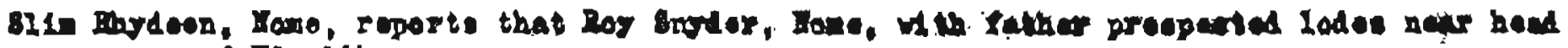
of Ilvails.

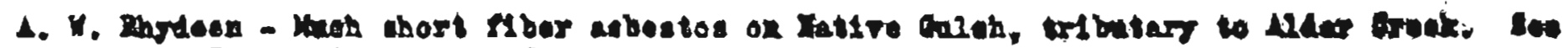
Thoma tequan of Oandla.

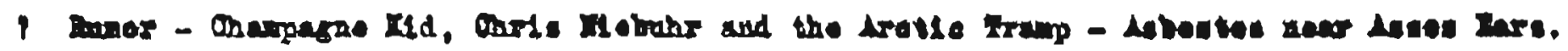

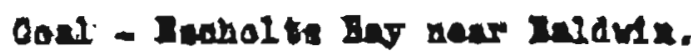

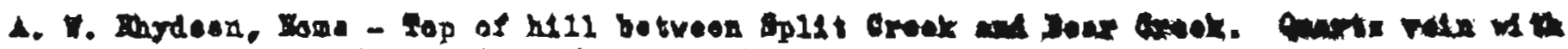

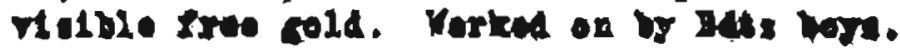

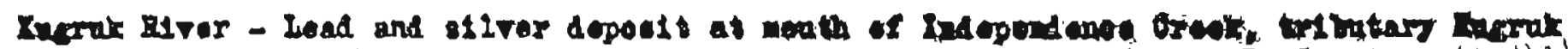

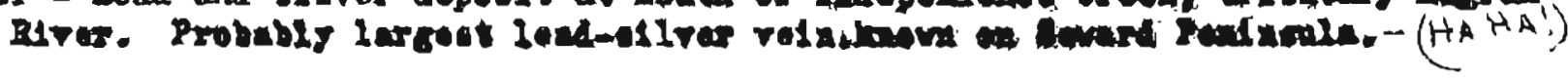

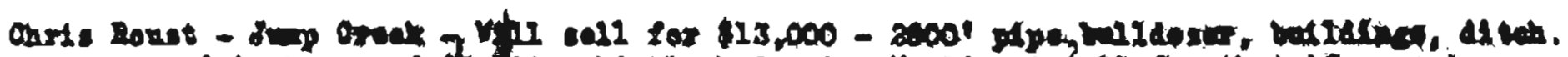

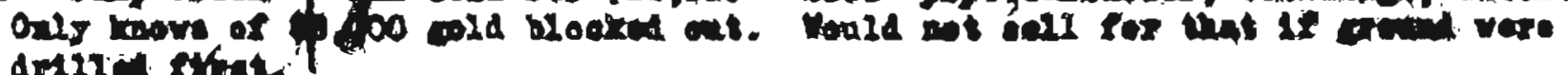

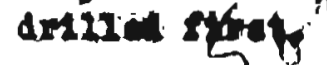

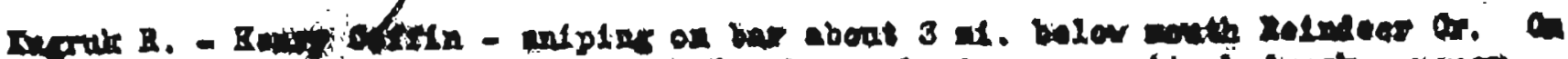

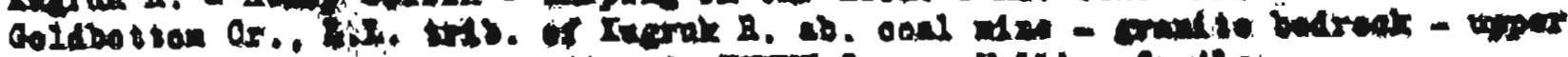

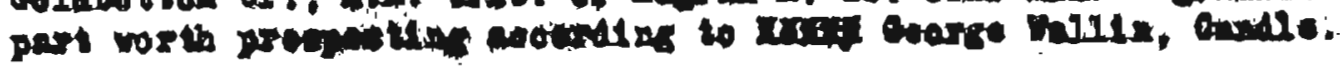

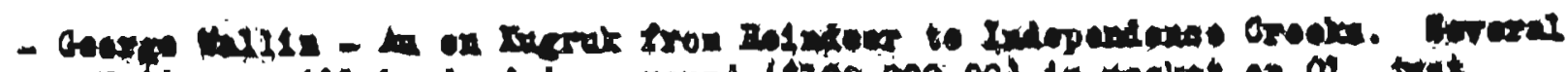

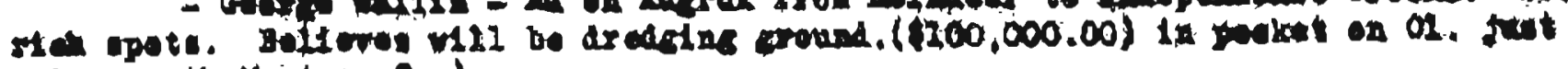

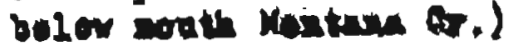

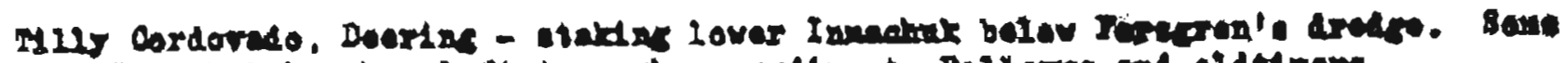

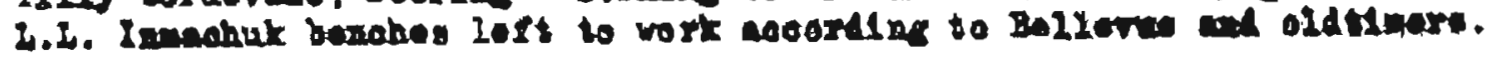

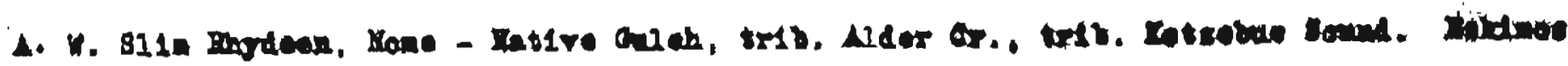
hore oulped there. 


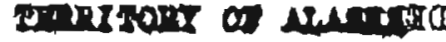 \\ Bepartiont of Xines}

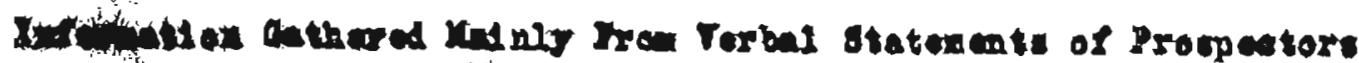
Mran 394.

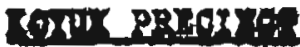

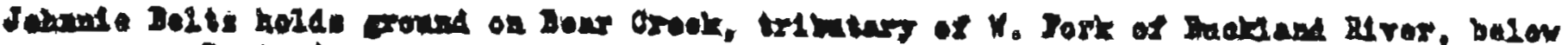

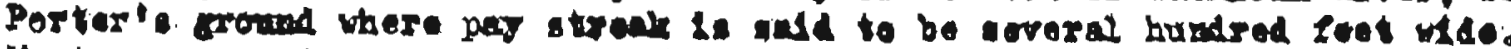

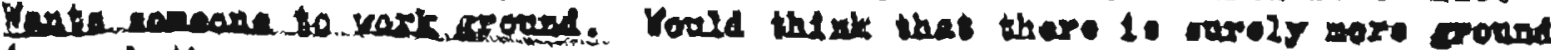
so work thare.

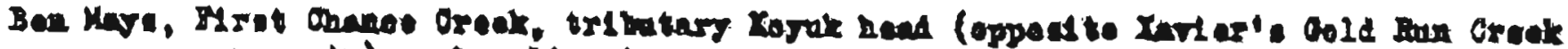
proparty) - charalieg in.

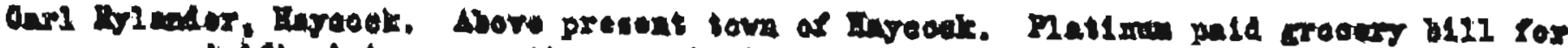

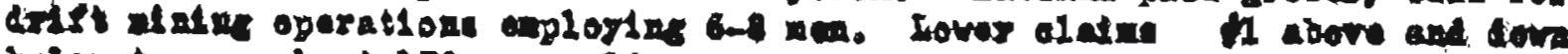

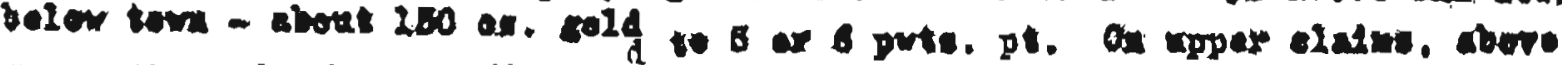

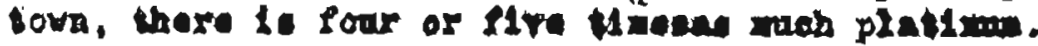

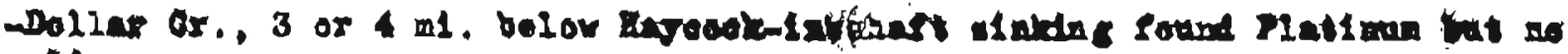
cold.

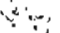

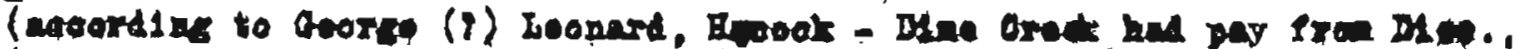

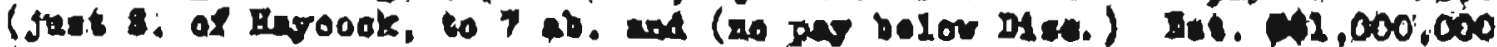

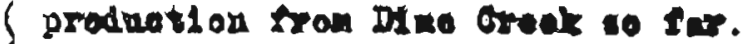

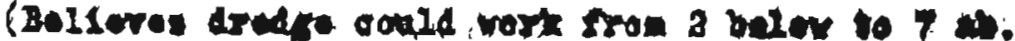

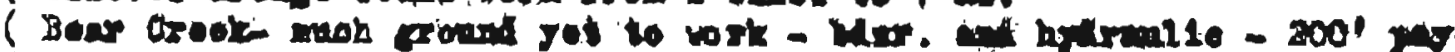

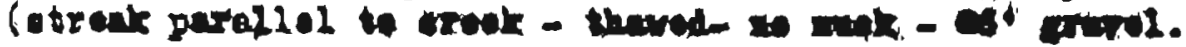

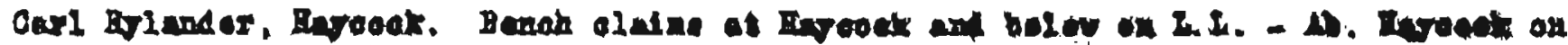

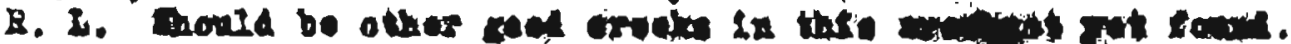

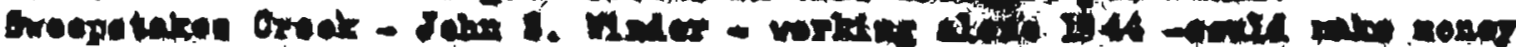

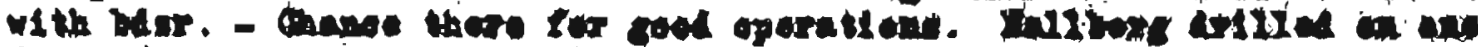

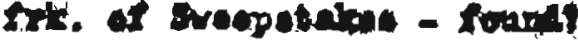

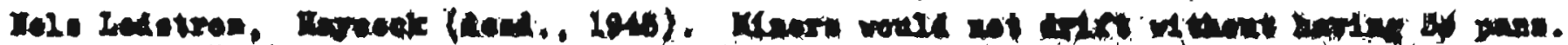

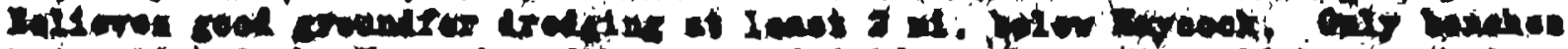

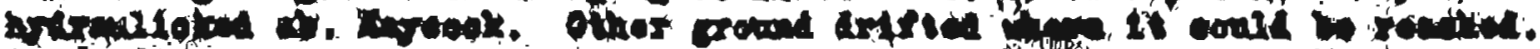

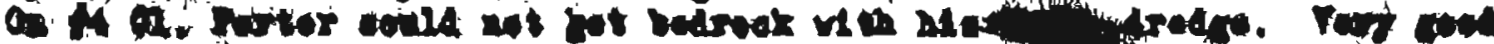

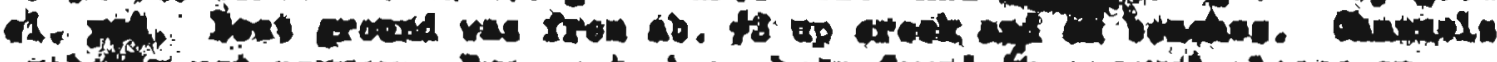

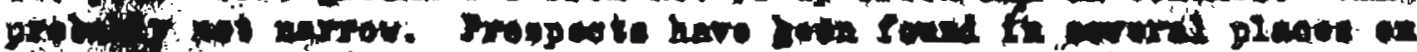

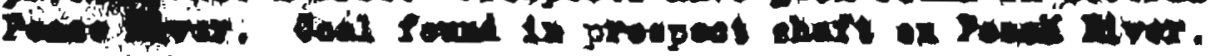

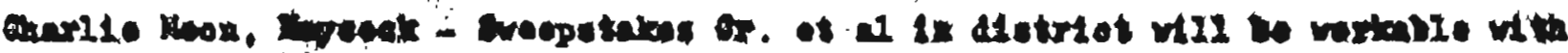
ancintinges. 
TURRI TOAY or ALASR

Dopartmont of Mines

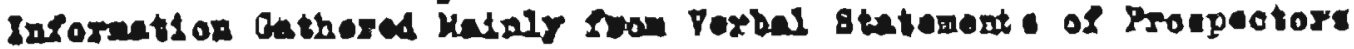
Merah, 1946

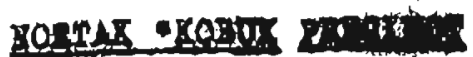

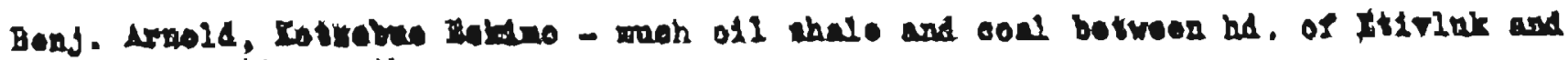
160 roult.

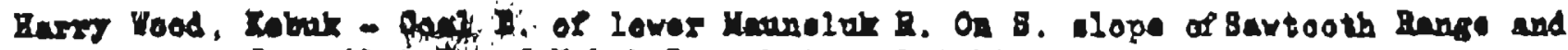

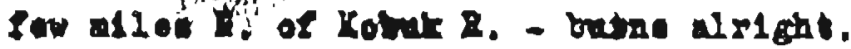

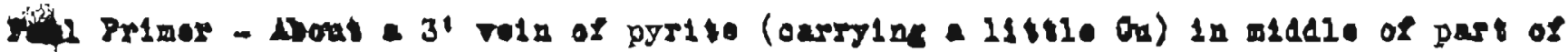
uppor Rogolartule onpron.

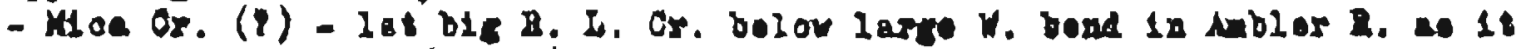

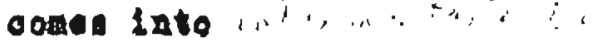

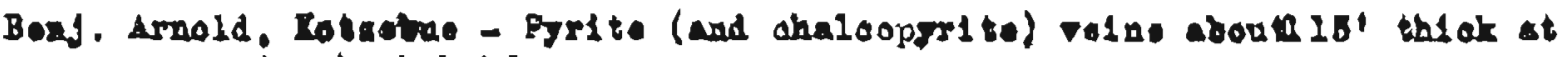
Lraingahol be.

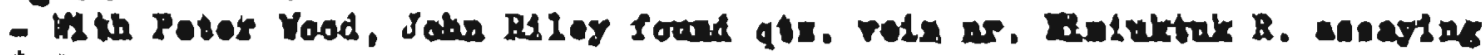
$\$ 32$ a ton.

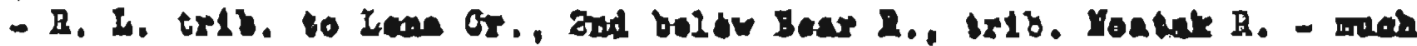

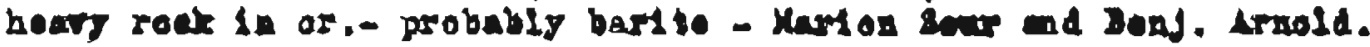

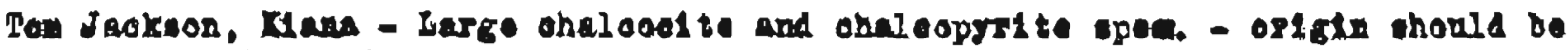
loarnod.

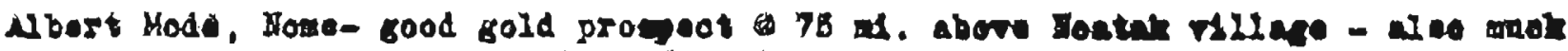

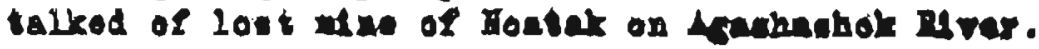

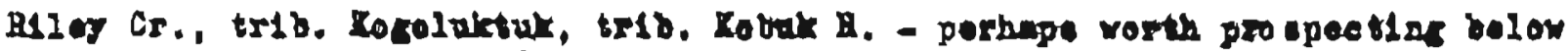
B1lyg Doogan's as well as at Doegre's.

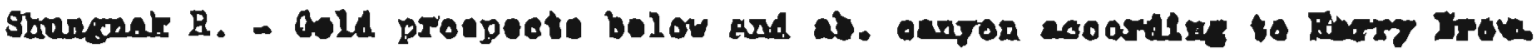

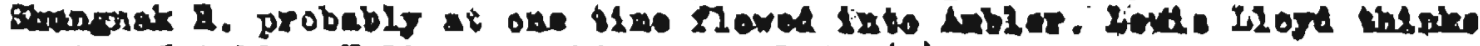
parts of latior Pallog abould be dzilled. (7)

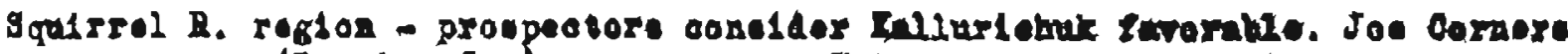

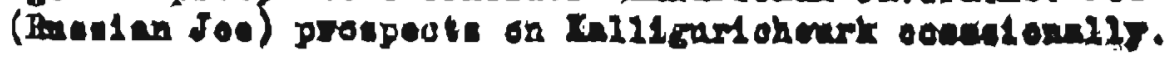

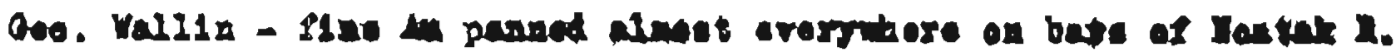

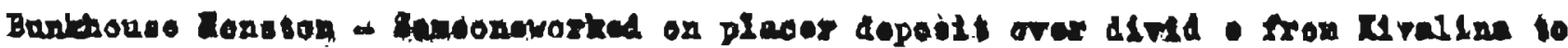

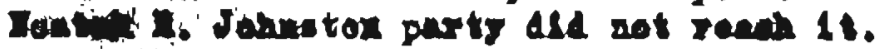

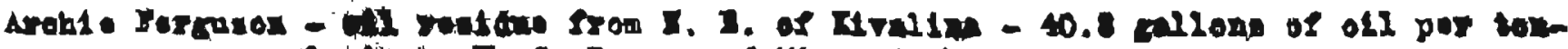

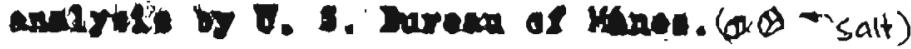

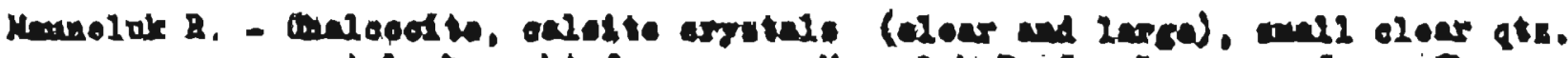

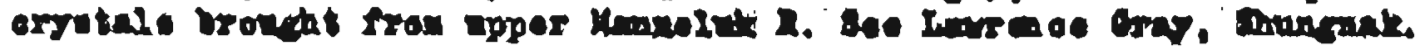

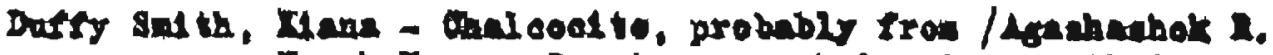

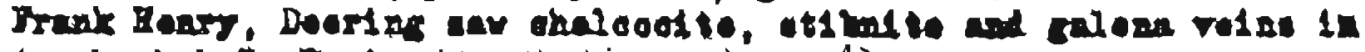

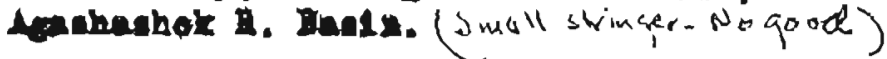


NOATAK * KOBUK PRECINCT (Cont'd)

Peter Atoruk, Kian - I. I. Noatak R. 25 mi. ab. Anluk mth. - dark yellow materlal, conslating of soft butter, plcked out of cliff by hand and burned - oozling from cliff.

Vincinity of Norutak L., hd. of Kobuk R. - Guy Moyer claims-

if -Hd. PQh R. - opp. Hog - prospectorg consldered good for Au, according to Howard Lyng, Nomo.

Anluk R. - Good sigy of mineralization (Dlacar) according to H. B. Blankenshlp - Lede accoralng to Paddy Watt, Nome - Qtz. boing up Ambre $R$, eccording to Cherbey Maxwell, Nowe. Accordins to Jim Cross, Aniuk no good.

Charloy Maxwell

- ? \$3 m1. Irom shore - 25-30 m1, notth of kotzobue -\$20 atz. voln- Robb1as knowe about.

- Canyon on Tutuksuk R. - glate formation - gaiena. Big veing froo roll1ing and bese ore on Tutukauk $15 \mathrm{mi}$. abore mth. Porbably base metal depost with littlo Ald.

-Billy Boogan ground - Blley Cr. - R. L. opposite hd. of Dahl Cr. - wteo gold placer and qtz. velng there - see Roed report "Uoper Kobuk Gold Molds"

Bull. 536 (p. 118, P. 146)

Jim Crosa, Ambler noar Redstone - (big boulders, never prospoctod, thawed goound - recovers very small amounts of modorately flue, flajg du. - Shungnak R., graes rooto down to 4' should make moneg .. more As than Bear Cr., Stirrel R. - Goin̈ up Klery over divide into Kallarichuk B. (flrat R. ab. Squirrol) good timber- posaibilitieg for Au very good there.

Archio Ierguson - Pb - vein on California Crl, trib. Kogoluktuk B., in canyon.

Iadac Jackson - Jack darnop Hana - blg Cu vein on cliff́ - Klana aroa.

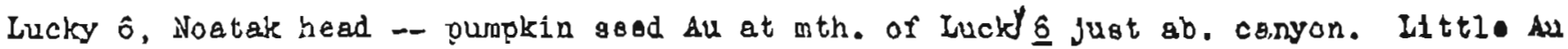
ab. Ist 2 clatme up frow moth. Bodrock concentration on natural Iffies each spring. Look for qtz. lead and bench according to Lewl B Lloyd.

ivoatak R. - elaing located. Gold lode, Charley Goodwin (15-16 m1. It, of Noatak v1llage) Soe placer - Loan Cr. - Yikuk Harris) Sours, Skin, Arnold)

\section{Main Creok Lod.}

Selion River - Good looking placer country from Nikok R. to abovo Kanaktok Crl, noar Jackson-Heral om base camp. However, 11 ttle du hag boen observed in $\mathbf{S}$ or in panning there.

J1m Cross, Ambler R., trib. of Shungnak R. - Hould 11keto prospect Redetone B. head. Bellever good qtz. on kunt R1ver. 


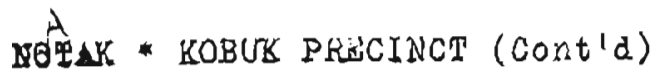

Harry Brown, Kobuk P. . Au has been found on Beaver Cr., a trib. to tho upper Kobuk R. Plck R., trbb. to upper zobuk from soutb - colors thru gravel - bedrock not reached.

Benj. Arnold, Kotzebue - casolterite nusetet picked uo in El1 R, Gravolo juat abovo _.. Leart $M t ., a b .8 \mathrm{ml}$. above Ell $R$. Lower nain fork.

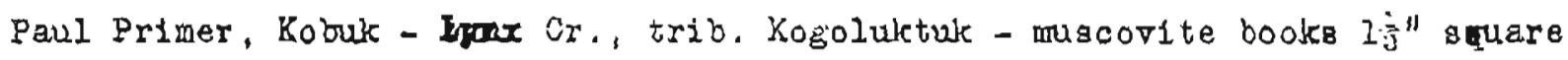

Red Glaggow - A.C.S. civilian (Nome) -r got 75申 pang on Chandlor R. from bar out of high mts bolow Chandlar lako 


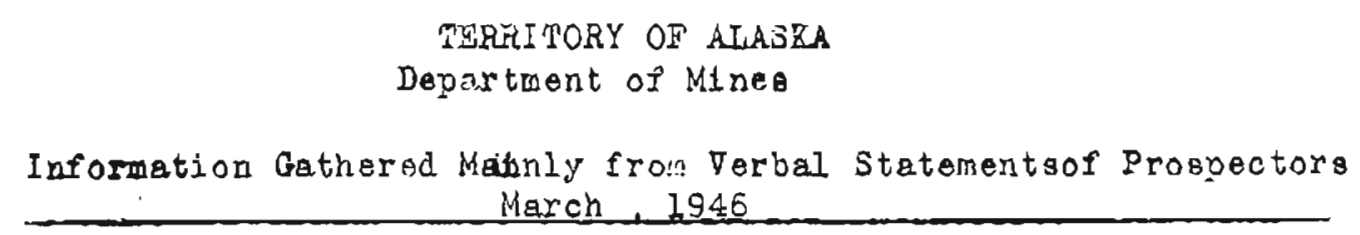

PORT ULARENCR -PRROINCT

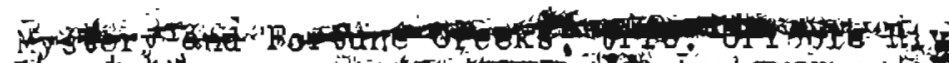

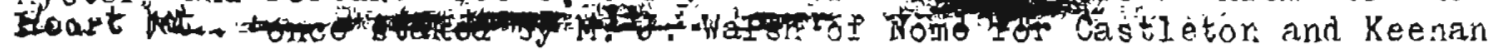

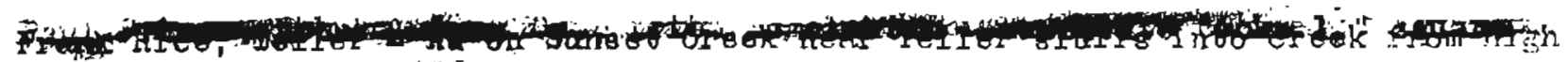
Denchea (crossut 3 beach channels, onowent better thon 7 oer pan at old Au prico) - gold in jenches is fine - several good areas for future orospecting.

Harvey Grant, Nome - Hgg Cr., trib. to Aglaouk - ainnaber lodo 1 California R. - tor in $6^{\prime \prime}$ vein exposed on mt. top. 1

Bluestone $R$. - Gold Run and other creeks in arag are good - Twoet fanlly et Tejler Is interested. (Offield $\mathrm{Cr}$. and vicinity near Teller.

Ot to Worm - Boulder Cr. - tin prospectg good enough for mall operator - egpectally near head of creek.

J. K. Johngton - Oll shale. "Shaly looking stuffl, found by netrea on Pish R. netives burned $1 \mathrm{t}$.

Portaje Or., trib. Anerican River, Senard Penin, - olnnebar pebbles ta in diemetor Avthur MeLain 


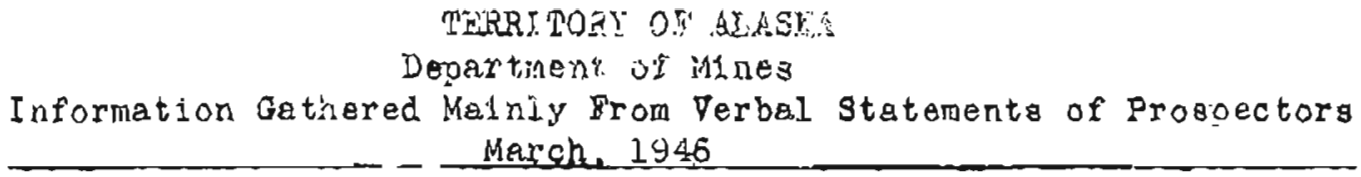

\section{D, MI U⿴AR PRECINCI}

Simon Segoonik, Ungelik - good look1ng creek trib. Shaktoulik opp. ha. N. Frk. of Unalakl eet?

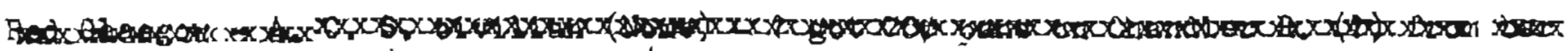

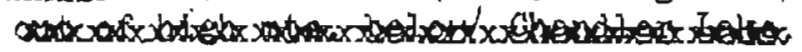

$?$ Fumor - large mice flakes in Rolndeer Hills - according to Ting Forshaug there is Erant te porphyry there

W. L. Adamo - hd. Andreafoki R. - Pb mineralization - supoosod to be 1 day walk from St. Michrol 
TERRI TORY OF ALASKA

Deoartment of Mires

Information Gathered Nainly From Verbal Statementsof Prospectora

March, 1946

Eskil Anderson

\section{WADENLAMPON PBECINOT}

P. Hiurm - Kotlik River, between Kotlik and hanilton - gold prosoects worth drilling

Creek near Marshall - investigate

W. L. Adame - bahind Russion Mission - several hundred feet tunnel - good proposition according to Brooks, U. S. G. B. - about $15 \mathrm{ml}$. 'W. and glightly N. from Antak $M t$.

- probably rafers to Cu-Au depos1t described in U. S. G. S. Bull, 622, po. 358-360 


\section{TISID DIVIBIONY}

Miliet, Junear -

-Bonanza (1) Cr.; thawed Eround; 37 per yd,; dredging ground; $40 \mathrm{mi}$. N. of mth. of Chulitne R., Lake Clark. Sank shafte which cared; brought in drill and left.

Valdez Cr. - lodes and placer - Bull. 849-H et. Al.

-Ungaf Is., Shunagins, hd. Cold Karbor, soep - Gl ot. asphalt baso oll a day dustín.

-Bench at mth. Big Bonenza and Chilikandostren - alco B1g Bonanza good placer district in that part of Mulohatna rogion - Bull. 655

Bull.773 - Kamishaik-Katwal - Millot Copper - NoNo111 group - doscr. 0. 173, Map p.160

Frank Reliy, Bx. 1268, Fhone Black 632, Junoau. Mica flakes, 4" clear, flat; shoets pasaed by G. Flectric -935 miles from canyon on liasks R. R. In unmeppod arsa on or near Dovil Croek, Bouth olde of atream.

Fred Wann - Whtte $R$. Alatrict - Ou veins $4^{\prime}$ to $5^{\prime}$ to 30 to $40^{\prime}$ zo nes Gejene - $30^{\prime}$ vein Gola - E. of Northray - $11 / 2^{1}$ veln - \$60 por ton Freclay - whito $R$, region 
TERRITORY OE ALASKA

Dopartment, of lines

Informstion Gathered Malnly From Verbal Statements of Prospector March $\$ 946$

\section{CHANDAZAR PRESINCT}

J. 1. Owens, Ft. Yukon - manganese and oll samples (1913-1913)

Harry Anthony, Ft. Yuron - hlgh grade bitumious coal

James Ward, Tt. Tlkon - brine from salt 1ake, $\mathrm{Na}$ and $\mathrm{KCL}, \mathrm{NaSO}_{4}, \mathrm{Na}_{3}$

H. B. Rodnan, Ft. Yukon - gal ena low in Ag

Ambroge William, Venetie - Cu and graphite specimens -Up Chandalor R., ab. is mi. ab. Little Squaw Lake, stlver galona 2030 oz.

Ag. per ton - emall atringers in limestone.

Baby Mte. N. W. of Chandalor Lake - qtz, district according to Denny OlIeofe

Denny O'Keefo, Wiseman - good Alu prospoots found at hd. of W. Trk. of Chandar.

Mike Hynton and A. W. Amero (Chandalar); also Charlie Schulta, Beaver - found froe Au in qtz. at ho. of H. Fork of Chandalar R. Hertie, USGS., sald to have reported $1 / 2$ oz. aseays.

Dennte O'keefe, Wlsemen - Baby Cr., R. I. trij. of Ojiandalar just ab. Chandalar L. qtz. district and some gmall placer. Velus up to $10^{\prime}$ thlck carrying scorodi to as Ifttlo Squaw arsa. El11s Anderson, Chandalar, sark ghapts there. - In Littilo Squaw -Big Crook area of Chandelar there apparentiy io nothilng left for new prospoctors.

According to Nels (Tiny) Forahaug - Lt. Morgan panned $\$ 2.00$ - 1 pan. On Okpllak or Canning R. and offers to pay his expenses to orospec t creok in 1845. story questionable.

Accordine to friend of Castel - Hd. of Seganarlktok R., - pay bo good All district /trib. Aretic Ocoan oast of Barrow.

Harry Knudeen, Barrow - Sank shaft $80 \mathrm{ml}$. Inland from Barter I. on Okpllak B. Slate bodrock at $20^{\prime}$ - fine gold concentrated thero - oncouraging reovite but did not go back.

Denny O'Keofo - O'Zeofe has much h1-grade qtz. from Chandelar d1strict - plcked samole brought $1 \bar{n}$ average several thousand dollarg por ton frec dus - Also found geveral creekscontalalng coarse Au - Showed coaree du frolo one crook. - Tolls about very largo lmperfect qtz. cryotals from Nolan $\mathrm{Cr}$., Koyokuk $\mathrm{R}$. 


\author{
TERRITORY OR AIASKA \\ Dopartment of Minos \\ Information Gathered Mainly From Verbal Statemento of Prospectots \\ March, 1946
}

\title{
FARBAIVSS PRBCINCT
}

T. Wolff - Totatlanika - Brosty Potter, Nels Jackeon. Placer claims -also Moose Cr. Eva Cr. - (Honestake Cr.l) Platto Cr. gmall lodes on California Cr. Moose Cr., Eva Cr., Spruce Cr.. Caribou Cr.

Mohowk Mine and Grant Mines, Ester Dome -- gtill good prosyects.

Dave Mutchler, Chene Hotaprings - Thinke machinery could be nut on Shamrock Cr. where voe Chesna tyes.

Lou Colbert - Floor of last level Claary Tunesten Mino - good lead for 200 ' on bottom lovel - Thingeten ore covers bottom of drift. 


\section{BORTYIIIS PRECINCI}

- Kotchumstuk Cr., - drillins 1942 - Gold and hy Cr. regions ghould oo prospected.

Johnile Pela -muacovito - Fairolay Cr., - 40 mile - 5" x 6"l sheetg - mioa on knoll wh thin 10 miles of creak.

Bruce Thomas - Michigan and Bear Croeks are worth drilling (40 Milo - trio Machigan, Grantte and Bear)

Whitehead - $40 \mathrm{MB}$. - Found good prospects on Middle Fork and on Eureke Frk. Also some on Denison Fork ( $\mathbb{E}$. Frk. ) In streams cutting intrusives shown on mao.

-Ci in otz. Voin botween W. Frk. of Ladue B, and Dentson Frk. See PI. 2, -Bull. 827, Juneals Offico.

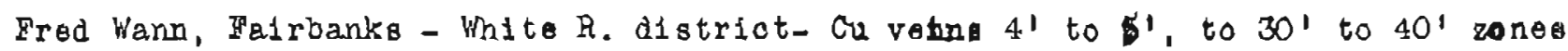
Galena - $30^{\prime}$ vein Gold - I. of Northway - $11 / 2^{\prime}$ vein- $\$ 60$ per ton Fireclay - Wht te B. region.

4. G. Wilcox - \$30 asaay from amall vein in Alcan Hiway cut W. of Northway

Bruce Thomag - Copper velne in Charley R. area. Still good du atz. In Ooodpagter. Pt. on Vulchatina. 


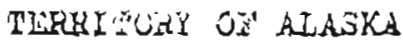

Departyent of Manes

Information Gathered Matuly From Verbal Statements of Prospectors March 1946

FORT GIBBON PRHCYNCT

Tanane District - Creek below walter Fher drilled by Bdds Farrel and Jobn Holiday I1 sher worked Granite Cr.

- Pb dejogit at base of high mt, on Moran Cr., sample ran $\$ 160$ a ton 


$$
\begin{aligned}
& \text { TERAIMOKY OF AYASKA } \\
& \text { Depentrisnt of MJ nes } \\
& \text { Information Gathered Mainly from Verbal Statements of Prospectors } \\
& \text { March, } 3946
\end{aligned}
$$

\section{HOT SPRIHGS PRLCINCT}

Accordlng to Gus Benson, Coma., Hot Sprlngs (Manley) - good areb for orogoecting with cat and drill between Eureke Cr. and Livengood. Also Boulder Cr. 


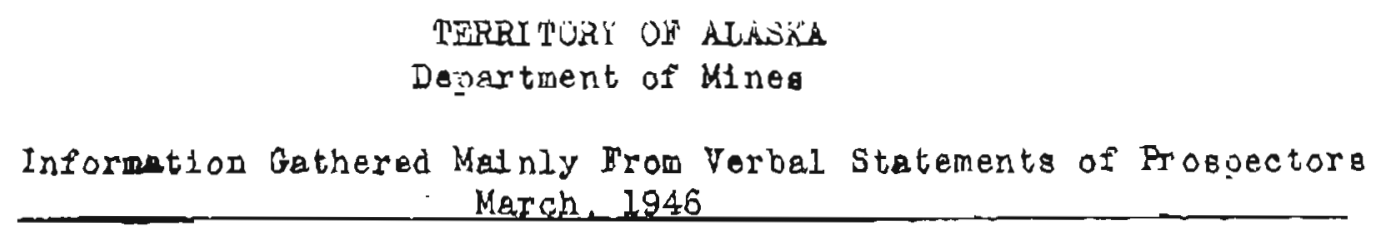

INNOEO PQRCINCT

Goldon Horn Mine - may still bo good inine, had very rich ore, gold in arsenozyrito, some say it can bo mined if satisfactory milling methoda are used.

20. 


\section{TERRI TORY OF ALASKA \\ Departinent of Mines}

Information Gathered Kainly Trom Vorbal Statements of Prospectors March, 1946

\section{KOYUKUK FAEEI ICT}

R. L, Stewart, Juneau- orospectora claim to have panned $\$ 10$ per day on hatr. crosks of Alatna $\bar{R}$. (MLtenburg Cr.?)

Charlle تvans and Jokn Beaton proapecting on $12 \mathrm{ml}$. Cr. (1944). Grans believes Alder or., hilng. Into hd. of ' $2 \mathrm{H}$. has enough monoy for thorough orosoecting. Alder $\mathrm{Cr}$. and others in Koyuluk are doep and wet. Should bo drilled.

Elmer Hayes- ilid Lake, Fake $\mathrm{Cr}$. , floot na,tive 31 and Cu, mitintod quartz, scheelits, chalcocite, large tourmallnesand cannel coal.

Otto Geist - uover Alatno - lergo Pb-Ag de joo1t (John Mecklenberg ,ietate - enormous AgPo ledge. Seo tirnie Johnson, Bettles, Brank Smlth, lild Lake)

Dennis Olyoefo - Nr, ha. Bettles Rl, on Hillow Cr., Mulo Cr., 8 Mile Cr. - Native Cu nuggets. Also argentite on $8 \mathrm{Mile} \mathrm{Cr}$.

Frank Theisen - 14 clains on Slate Cr. (being dr1lled by Ropo, 1945) - Ollver Gaapelle al 80 has claims - With Jack Allen wont up Alatas - considerod prospect too inacesalole.

Donnis OlKeefe, Kiseman - Archibald - may bo worked by anal I machinery Hammond R. )

Nolan Cr. ) Posalble drodglag prop. mramway Bar )

\section{- RodA Roport}

Slat Cr, and Hmme Cr. - small meohinery.

Stannich Bro日. - Porcupla Cr.

Twelvomil Cr. - machinery

Lake, Spring, Jay and Ryo Cr. - Mid Lako area. 


TERRI TORY OF ALASKA
Department of Mines
Information Gatiered Mainly From Verbal Statements of Prospectors
March. 1946

KUSEOKYIIV PRECINCT

Frank Roder - numerous cinnabas prospects at hd. of Oskainalik R. (betweon Aniate and Holitna R.).

- Ag-Po prosiect up Oskawalik $\$$

.. - Stibnite va1n ab. 60 mile from Napemute acrosg river and im ame general erea as other two.

Wilson Cr., trib. George R. - Georgetown viclnity - Kugkokwim R. - 36 per pan in Alu according to H. G. Hilcox, College. Other small atrearne In Kuskokwim should be arospected.

Jay Cook- good ground on upper Salmon RI, trib. lower Kuskokwim, on creek heading into Fisher Dome (B.I.T.)

Bruco Thoma - blgh dg head Stony R. - algo Au lode

-Placer and lode possibilitieg good at hd. of Stony and S. Trk. of Kugkokwim. A. E. Wal gannen in area unmanoed on Bull. 862. 


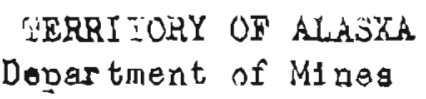

Informetion Gathered Madnly From Verbal Statements of Prospectors March, 1946

IiT, MCKINLEY PRECINCT

Nixon Forke Mine, Bull. 738, p. 128 Bull. 864-0., p. 229. 
TERRITORY OF ALASKA

Dogartiont of Mines

Information Gathered Mainily From Verbal Statements of Proapectors March 1946

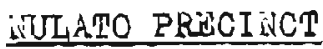

Perseverance Lode, Kaltek hills - Ag-Fy lode near Galena, 225 tons h1-gradie ahipoedunstaked - said to be sohalertte vein there also.

$$
\hat{6}:
$$

31rch B., Ruby district - Sald to be good dredgling gosstb1l1ty. Fart owned by Gus Uotila.

McDonald Cr., hawater, tributary of Anvik R. - $40 \mathrm{m1}$. S. I. Unalakieot - restaked many times with other creeks in that area. Some progpectorg made $\$ 2.00$ to $\$ 5.00$ a day shoveling- in.

Charley Tryck, Ruby - Agboatos ab. $50 \mathrm{ml}$. up YOko R, noar hd. of H. Frk., close to river on R. I.

- Also sad to be large chromil to deposit in samearsa. Platinum apparently was found $1 \mathrm{n}$ sare area (It's reported ldentification not coulutetay ver1fied.)

Gold prospects also seid to have boen found on Nowitna trib. - Henry Biliman took $\$ 5000$ out in one $\mathrm{yr}$. (Probably Cailfornie Cr., trib. Totne, trib. Nove (Nowitne.)

A. H. Amero, Chandalar- on Mrib. of Alatne halng. againgt middle portion of Luokg $6 \mathrm{Cr}$. (also creek adjacent to it). Fnormous base oro doposit (\$12.80 ver ton in $\mathrm{Au}$ ) 


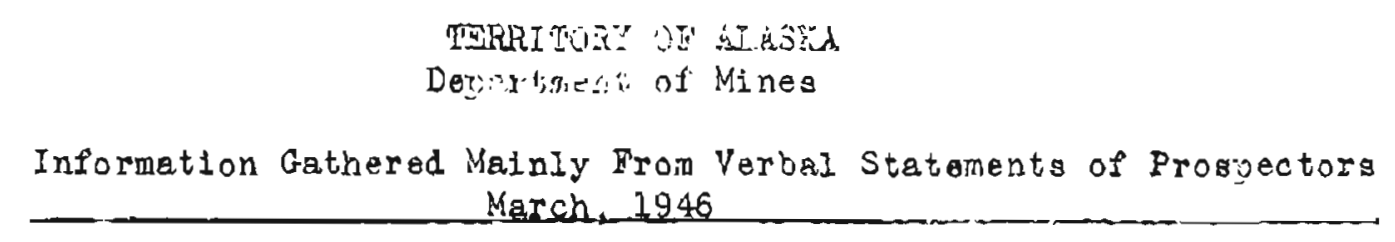

\section{RAMPART PRECINTCT}

Henry Luttman - Asbertos (chrygotile and omphioolo) on Droamlani Cr. - 6 claj ins staked as on Aobestos Gulch, trib. Blg Salt Cr. (1925). N. of Yulon and Ramport 20 to 35 miles.

Fay R., trib. to Yukon from north aoove Ramoart - bot goringa, two, about 8 miles apart and about 40 inlles above mouth of Ray $R$.

Charley Swanson - Hodzana R. region - Au mining 1944

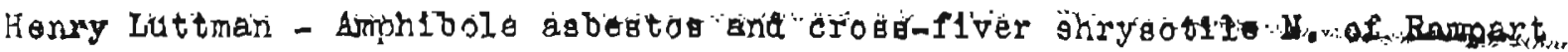


TERRI TORY OF ALASKA

Department of Mines

Information Qathered Mainly From Verbal Statemente of Prosoctora March. 1946

\section{KOUGAROX PBÜCINOT}

According to Pete Hiurm, Nome, - should drill Winter Creek, opy. há. Winòy Crook.

(Good Au grall drodging posaibliles on upoar Quartz $C_{r}$, trio. Lougarok) Proapects on flat about $1 / 2 \mathrm{ml}$. from Shelton curving around bass of Coffee Dome towerds Doffee Dr., (Good atz. lead on Camp Cr., trib. Coffeo Cr., trio. Kougarok)

Most ground on Coffee Cr. is patented. Grant Mining Co. has all of upoer creek exceot elain worked in Aug. $144 \mathrm{mth}$. of Baglo Draw, owned by Pete Hiurm ot al.

- Eagle Draw and Wonder Gulch have good money but little R2O. - benohea on Coffeo Cr. should do prospected.

Loule Nashenweng, Nome - Quartz Ce., - according to L. Nashonweng - erery trib. to Uoper Qtz, has oay streale- many bench channolo must be thero but have not been discovered- expects creek to bo worked for 50 jears at present rate. Nashenweng and Rasmus have most ground on Qt $z$. proper. Pay streak rich on $\mathrm{N}$. frk, unworked, only lower 2 or 3 claims staked. Joe Cr., worked by Charley Jager, rich, could make money if worked differently. Jager works l no. each $7 \mathbf{7}$. - over $\$ 1000.00$ by hand methods during some of there yeare.

Fiximos sniped on upper Checkera Cr., probably open - may be pay on upper Checkers $\mathrm{Cr}_{\text {. }}$.

Neshedreng has very rich ground and wath of 1t on Qtz. Cr. mear mth. of Dahl Cr.

Little prospecting done opp. hds. Dah1, Coffec and Qtz. Crooks.

Indepondence $\mathrm{Cr}$., trib. to Qtz. Cr, has pay gtreak and $1 \mathrm{~s}$ not all ataked. Qtz. Cr. - 55 per yd. from Checkere cr. up. Thawng only difficulty in Hay of dradgo.

For straight prospecting - wahk same as on Dahl Cr. 1 a croeks draining N. W. sloper of Beddolsben Mt. - streame practically unprospeotod. Should prospect with drtll acrose flla, Birch, oto. - crobs-cut $2 \mathrm{cl}$. longht ab. omergonce of creokg from footh1118 - 19 no prospects more on.

Upoer Xougarok - iacomphete information. Soe M. J. Wal ob According to Wal sh - no untaken known dredge sound loft.

Otto Worm and Bourgault - Bd Haven's ground on Coffee Cr. Was taken over by Herrey Grant in 1945. Could probably take in $\$ 10,000$ I $5 \mathrm{r}$., two mon. Lowar and best according to B1II Bourgault. Garvey Grant thinks there will oe money for Otto Worm on Windy $\mathrm{Cr}$.

Harvey Gratt- orown, dead-looking tundra with alkalt ataln, markg old Channels in Kougarok datrict (Low saddle from Joo Cr. over to N. Frk. should wark old channel) 
KOUGAROK PRECINCT (con't)

Pete Wiurm - Coffoe Cr., much broken up quartz - Log Cabin Gulch - free Au cominon In this qtz. 